Inteligencia Espiritual y Valores Personales en los integrantes de la Coordinación del Proyecto Educativo Regional.

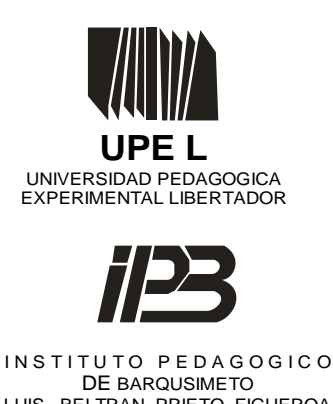

DE BARQUSIMETO
LUIS BELTRAN PRIETO FIGUEROA



Órgano Divulgativo de la Subdirección de Investigación y Postgrado del Instituto Pedagógico de Barquisimeto “Luis Beltrán Prieto Figueroa"

BARQUISIMETO - EDO. LARA - VENEZUELA

NUEVA ETAPA

FORMATO ELECTRÓNICO

DEPOSITO LEGAL: ppi201002LA3674

Volumen $18 \mathrm{~N}^{\circ} 1$

ISSN: 2244-7296

Enero - Abril 2014

\title{
INTELIGENCIA ESPIRITUAL Y VALORES PERSONALES EN LOS INTEGRANTES DE LA COORDINACIÓN DEL PROYECTO EDUCATIVO REGIONAL
}

SPIRITUAL INTELLIGENCE AND PERSONAL VALUES IN THE REGIONAL EDUCATIONAL PROJECT COORDINATION

\author{
Carmen M. González. * \\ Luis Vera G. **
}

* Escuela Antonio José Urquinaona. Santa Rita estado Zulia ** Universidad Privada Dr. Rafael Belloso Chacín ( URBE) 


\section{INTELIGENCIA ESPIRITUAL Y VALORES PERSONALES EN LOS INTEGRANTES DE LA COORDINACIÓN DEL PROYECTO EDUCATIVO REGIONAL \\ SPIRITUAL INTELLIGENCE AND PERSONAL VALUES IN THE REGIONAL EDUCATIONAL PROJECT COORDINATION}

\section{TRABAJO DE INVESTIGACIÒN}

Recibido:10-02-14

\section{RESUMEN}

La finalidad de la investigación, fue proponer lineamientos estratégicos para fortalecer la inteligencia espiritual y los valores personales del personal de la Coordinación del Proyecto Educativo Regional en el Municipio Santa Rita, estado Zulia. La metodología fue cuantitativa el tipo de estudio fue proyectivo, con nivel descriptivo correlacional, el diseño fue de campo, no experimental, transaccional; la población se constituyó por directivos, docentes, obreros y administrativos. La técnica de recolección de datos fue la encuesta y el instrumento un cuestionario con escala tipo Likert, sometido a validez y confiablidad. La técnica para el análisis de datos fue la estadística descriptiva y el coeficiente de correlación de Pearson. Los resultados permitieron elaborar lineamientos con base en los seis caminos de Zohar y Marshall (2001), como: el deber, amor y entrega, conocimiento, hermandad, entre otros, que brindan bases sólidas para que actividades personales y profesionales se realicen en atención a las necesidades.

Descriptores: Inteligencia espiritual, valores personales, proyecto educativo.

\author{
Carmen María González Guerra* \\ Escuela Antonio José Urquinaona. Santa Rita estado \\ Zulia. \\ Luis José Vera Guadrón** \\ URBE \\ Aceptado: 12-04-14
}

ABSTRACT
The purpose of this study was to propose strategic guidelines to reinforce the spiritual intelligence and personal values in the Regional Educational Project Coordinators in Municipio Santa Rita, Zulia State. A quantitative projective study with correlative descriptive level, and a field, non experimental transactional design was followed in this research with principals, teachers, workers and administrative personal in different institutions of the State. A questionnaire with a Likert scale model was used to collect the data. Data was analyzed with descriptive statistics and a Pearson correlation coefficient. Results helped to elaborate guidelines based on the six ways of Zohar and Marshall (2001), such as duty, love, commitment, knowledge and brotherhood that provides solid foundations for personal and professional activities to be performed based on needs.

Keywords: spiritual intelligence, personal values, educational project

\footnotetext{
* Doctora en Ciencias de la Educación. Universidad Privada Dr. Rafael Belloso Chacín (URBE). Docente de la escuela Antonio José Urquinaona, secretaría de educación de la gobernación del estado Zulia; carmengonzalez.guerra@gmail.com

** Doctor en Ciencias de la Educación. Investigador acreditado por PEII. Nivel B, convocatoria 2013. Comité Académico Doctorado Ciencias de la Educación. Línea de Investigación Docencia y Currículo. Coordinador de la línea de investigación Formación Docente Universidad Nacional Experimental Rafael María Baralt; luisveraguadron@gmail.com
} 


\section{INTRODUCCIÓN}

La sociedad experimenta desde hace décadas avances científicos, tecnológico y el crecimiento de grandes ciudades, evidenciando la conquista de la naturaleza, controlando de esta manera el mundo exterior, dejando en un segundo plano el desarrollo humano interno y espiritual. En relación a lo anterior Rodríguez (2012) desde la UNESCO expresa que al ser humano lo han dejado solo, la ciencia le ha dado bienestar y maravillas tecnológicas, lo ha acercado al futuro, pero lo ha alejado del presente. En consecuencia, se presentan múltiples situaciones conflictivas en todos los contextos y por ende en las instituciones educativas, en tal sentido se plantea el análisis de la inteligencia espiritual, puesto que se hace necesario un cambio de paradigma en la práctica docente para obtener seres humanos integrales.

En la actualidad, existe gran cantidad de información relacionada con la inteligencia espiritual, pues, según Zohar y Marshall (2001) "es con la que afrontamos y resolvemos problemas de significados, valores y con la que se puede poner los actos y la vida en un contexto más amplio, rico, significativo y determinar el camino vital más valioso” (p.19), esto es importante, porque la humanidad se encuentra sumergida en el consumo para la complacencia sensorial, producto de una cultura carente de sensibilidad espiritual, donde prevalece el cuerpo - mente, y el placer de los sentidos.

Uno de los problemas que afecta al sistema educativo a nivel mundial, está relacionado con la falta de valores de algunos de sus directivos, docentes administrativos y obreros, impidiendo ello el logro de las metas propuestas, es decir, formar un individuo integral útil a la sociedad; aspecto que ha incidido negativamente en el quehacer administrativo y pedagógico, pues los mismos hacen alusión a las cualidades morales de dichos actores dentro del ámbito en el cual laboran, referido específicamente a sus acciones, características personales o sociales para asumir los cambios que se generan actualmente en dicho sistema.

Desde esta perspectiva, la organización escolar en América Latina desde finales del siglo XX, ha venido enfrentando el reto de asumir los diversos cambios que se han presentado en el campo científico, humanístico, tecnológico, político y social para poder sobrevivir en un ambiente de constante transformación. Esta situación por su dinamismo, 
ha generado la necesidad de formar un recurso o talento humano, capaz de asumir y afrontar con éxito las crecientes exigencias de la sociedad actual.

En este sentido, Buzan (2005), señala que cada vez hay más personas en las sociedades opulentas "que se están cansando de la superficialidad de sus vidas materialistas, buscan un nuevo conjunto de valores por los que vivir”. (p.21). En otras palabras, desean enfatizar un nuevo sentido de realización colectiva frente a una sociedad en un estado de creciente fragilidad. Se desprende de lo anterior, que el ser humano se aboca cada vez con mayor frecuencia a buscar otros medios no materiales para sentirse satisfecho en todos los ámbitos donde se desenvuelve.

En efecto, la armonía y la paz interior son fundamentales para que pueda sentirse bien emocionalmente, controlarse, manejar sus sentimientos, saber qué sentir, hacer y cómo actuar. Esto es posible, si espiritualmente se encuentra bien consigo mismo, por cuanto explica Covey (2005), "poseemos un paradigma incompleto de quiénes somos, de nuestra concepción de la naturaleza humana.” (p. 36). Es así como en la actualidad, es notorio hablar de inteligencia emocional pero más allá de ésta, se encuentra la espiritual.

Cabe destacar, que cada individuo posee en su interior el poder de conquistar la felicidad anhelada, solo hace falta conocer el camino, el cual consiste el alcanzar un nivel de evolución de la conciencia que le permita actuar desde su inteligencia espiritual, la cual, según Buzan (ob. cit), "permite ver el lado menos sobrio y más cómico de las cosas y recobrar cualidades como el entusiasmo, la alegría, la energía y la persistencia que caracterizan a los niños”. (p.14). Esto es así, porque incrementa la paz, fortaleza interior, ayudándolo a ser capaz de controlar o reducir las tensiones propias del ámbito laboral donde se desempeña.

En este contexto, resulta interesante destacar, que en el presente se define al individuo, hombre y mujer, como un ser biocéntrico, por el enfoque determinante de él como parte de la naturaleza y el ambiente donde se desenvuelve, el cual puede generar acciones cónsonas con el bienestar propio y del colectivo donde está inmerso. Esto viene dado por un desajuste detectado entre el sistema educativo tradicional y el modo de ser del individuo actual, lo cual genera obstáculos para dar a la educación su verdadero cometido, la formación de un individuo que provea y propicie su aprendizaje tomando en cuenta la 
constante y armónica acción del ser, saber, hacer para convivir, desde un punto de vista integral.

Por esta razón, los sistemas educativos se esfuerzan por darle mayor relevancia a la formación de los estudiantes, partiendo del ejemplo o modelo que observan en su ambiente escolar, donde los gerentes y docentes transmiten con su comportamiento lo que debe hacerse, basándose en lo correcto, es decir, en el respeto, la obediencia, la responsabilidad, la igualdad, solidaridad, libertad y paz, en procura de la armonía de cuerpo, mente y espíritu.

Para ello, directivos, docentes, administrativos y obreros deben dar cumplimiento a funciones específicas, referidas al papel que desempeñan durante el trabajo que ejecutan. En este caso, deben ser orientadores, evaluadores, coordinadores, asesores, apoyándose en la ejecución de las actividades para la integración y clarificación de valores personales, a fin de ser verdaderos promotores que contribuyan a las metas de la escuela, de la sociedad, tanto desde el punto de vista individual como colectivo.

En este orden de ideas, Barroso (2005), expresa que los valores son "creencias, principios intrínsecos que proporcionan un encuadre ético a la organización y al negocio”. (p.98) Sin embargo, la comprensión de valor depende de quién lo percibe. En otras palabras, el personal de las escuelas deben tener una visión clara y conocida de lo que quieren conseguir sus escuelas, tener una visión centrada en sus miembros, intereses y necesidades que responda a la honestidad en la transacción, lealtad con el cliente, compromiso con lo pautado y respeto al otro.

De igual manera, el personal que labora en las instituciones por su rol modelo de la organización educativa, debe contribuir al desarrollo global de cada persona: cuerpo, mente inteligencia, sensibilidad, sentido estético, responsabilidad individual y espiritualidad, por cuanto, todos los seres humanos deben estar en condiciones de dotarse de un pensamiento autónomo, crítico y elaborar un juicio propio, para determinar por sí mismos qué deben hacer en las diferentes circunstancias con las que se enfrentan en el ámbito laboral.

Por estas razones, el personal de las escuelas tiene el desafío de desarrollar acciones sustentadas en valores, de allí que la transversalidad aparece representando una esperanza 
para la recuperación moral en las Instituciones educativas, ya que se constituye como el eje para la solución de conflictos organizacionales. Dicho de otro modo, el personal tiene el deber de proporcionar vivencias de orden diverso a los alumnos y demás miembros de la comunidad educativa para tratar de promover aprendizajes y desarrollar capacidades también de naturaleza diversa, que coadyuven a su educación integral y no sólo a su instrucción. Por tanto, es importante indicar que la inteligencia espiritual está ligada a los valores humanos, pues no se puede separar la inteligencia del amor, la tolerancia, el respeto, la gratitud, la compasión, la honestidad y la solidaridad, que son la base para desarrollar y fortalecer los valores propios de cada organización.

Lo antes expuesto podrían ser las causas de la realidad que el personal experimenta en las instituciones donde solo se remiten al trabajo administrativo y pedagógico disponiendo poco tiempo para la convivencia con el personal, de allí que trae como consecuencia, relaciones interpersonales poco armoniosas, además de una comunicación limitada específicamente a lo laboral, obviando al ser humano en su espiritualidad y en su aspecto socio afectivo.

Esta crisis de valores, ha transitado durante mucho tiempo en las instituciones educativas, que de seguir así, terminarán convirtiéndose en centros donde no exista tolerancia y comunicación entre sus miembros, a pesar de que a los integrantes se les ha impartido algunos talleres sobre crecimiento personal y profesional. Es urgente entonces, ocuparse del vacío espiritual del cual padece el personal de estas instituciones.

Por tal motivo, se consideró necesario realizar una investigación orientada a proponer lineamientos estratégicos para fortalecer la inteligencia espiritual y los valores personales de los directivos, docentes, administrativos y obreros en la Coordinación del Proyecto Educativo Regional. Así como; identificar los principios de inteligencia espiritual; describir las habilidades de inteligencia espiritual; determinar los valores terminales; identificar los valores instrumentales y por último; establecer la relación entre la inteligencia espiritual y los valores personales de los integrantes de la Coordinación del Proyecto Educativo Regional.

En este sentido, es indispensable examinar e implementar tácticas, estrategias o algún mecanismo que ayude a mejorar las relaciones interpersonales y la labor en la institución, 
utilizando la inteligencia espiritual para generar un cambio significativo en este contexto globalizado, y así formar una gran comunidad donde se pueda apoyar, cuidar y fomentar el amor, la bondad, entre otros beneficios.

\section{ARGUMENTACIÓN TEÓRICA}

\section{Inteligencia Espiritual}

La inteligencia espiritual rige el orden innato e inteligente del universo, trasciende conceptos, costumbres y creencias, pues según Millman (1995) “forma la base de la moralidad humana” (p.5). Por su parte Gallegos (2007) plantea, que lo interesante del concepto de inteligencia espiritual, es que es aplicable a todas las áreas del hombre, por ende en el ámbito educativo, donde se reconsidera la única manera de construir una educación realmente integral en búsqueda de poder solventar la crisis de valores por la cual el mundo transita.

En este sentido, resulta importante resaltar que la espiritualidad no es usualmente entendida en palabras de Gallegos (2005) de manera correcta, debido a que es experimentada como orden interno total, como ausencia de conflicto, como amor universal y libertad incondicional, trasciende al ego y lleva a la persona a interesarse por el bienestar de todos los seres y no solo en el propio. Del mismo modo señala: "la inteligencia espiritual, es la inteligencia de nivel superior, que incluye y trasciende a la inteligencia emocional y la inteligencia intelectual”. (p.9).

Sin embargo, la espiritualidad ha quedado reprimida, fuera de los sistemas educativos; pero si realmente se quiere formar al ser humano integralmente y mediante la educación promover su desarrollo para que la sociedad pueda solucionar problemas con la perspectiva de pensar globalmente y actuar localmente, es necesario reconocer que en el aprendizaje existe una dimensión espiritual, además de la cognitiva, social, emocional, estética y físicacorporal, el cual debe ser extendido primeramente a directivos y docentes para luego ser llevado a todo el colectivo escolar.

Por lo tanto, desarrollar la inteligencia espiritual en el personal, lleva más allá del simple entrenamiento racional, afianza las bases del amor universal y hace entender que el desarrollo intelectual no representa ser mejores personas, mas compasivos, más honestos o 
más felices, es necesario que el conocimiento tenga además una dirección moral-espiritual que fomente estados de paz y armonía en las escuelas.

\section{Principios de la Inteligencia Espiritual}

Los principios de la inteligencia espiritual forman parte esencial del quehacer diario del docente. Al respecto Gallegos, (Ob.cit) señala "existen principios que permiten entender cuál es su naturaleza y cómo funciona; principios simples para encontrar en este mundo difícil, la paz interior” (p.5). Estos principios ayudarían a directivos, docentes, administrativos y obreros a ser mejores personas en el ámbito laboral educativo.

Atendiendo de manera precisa a estos principios de orden filosófico, se puede observar que la inteligencia espiritual es una realidad superior, y al ser fuera de lo material se trasciende, traspasa al ego, los apegos, pues es universal. Por lo tanto, cualquier ser humano puede encontrar su paz interior y superarse a sí mismo, pero es indispensable habilitar esa espiritualidad que solo puede ser captada mediante la contemplación. Tales principios se describen en los siguientes términos:

\section{Fortaleza del Amor}

Las personas por lo general, responden con sensaciones que les agradan o no, ante las situaciones que se le presentan en la vida cuando éstas se manifiestan continuamente o se evocan mediante la imaginación, estableciendo un afecto y el amor. Por lo tanto, el ejercicio constante de acciones tendientes a provocar respuestas afectivas agradables en el colectivo escolar, es una responsabilidad primaria y un principio de la inteligencia espiritual.

Según López y Violi (2001), el clima de cordialidad se logra únicamente con el ejercicio del amor, aumentándolo y consolidándolo. Sin embargo, se debe diferenciar la acción del afecto con la de la autoridad, pues ello destruye los grupos al bajar su autoestima. Cabe destacar, que el binomio constituido por la autoridad y el afecto, es una clave esencial en el funcionamiento de las instituciones educativas, es decir, su conocimiento y ejercicio continuo permiten el desarrollo de un grupo más comprensivo, por ende, más sano. 
En tal sentido, la actuación de los integrantes de las instituciones educativas resulta clave, por cuanto ellos son el modelo a seguir por los alumnos, dado que éstos realizan funciones para desarrollar el amor a través de sus orientaciones o mediante todas las prácticas laborales, lo que significa que pueden cambiar las actitudes en forma positiva, aunque también pueden crear barreras al establecer los cambios adecuados.

Al respecto, Ramos (2005), señala el amor es libre, se transmite solo en forma efectiva “cuando el sujeto lo adopta, lo vive conscientemente, fortaleciéndose en la convivencia diaria a través de las interacciones con los demás, de las experiencias que proporcionan la satisfacción y la alegría de vivirlo.” (p.66). Esto implica tomar en cuenta a la escuela, puesto que ésta, es una de las instituciones que más influye en el desarrollo humano, y la importancia de dar al amor plena vigencia en el ambiente escolar.

\section{Promoción del Bien}

De acuerdo con Gallegos (2006), el bien es la capacidad de desplegar la conciencia a niveles universales de donde se desprende un orden interno que permite al individuo darse cuenta que todos los seres están unidos, es un nivel en el cual el amor se encuentra estrechamente ligado a la inteligencia "que nos invita a actuar con honestidad y respeto, siendo compasivos, es decir, interesados incondicionalmente en el bienestar de toda la humanidad”. (p.48). por lo tanto, el individuo debe buscar el bien común a través de la experiencia, como expresa Verdú (2008), que el hacer el bien responde a una virtud.

\section{Actitud hacia la Paz}

Con respecto a este principio, Gallegos (ob.cit) "no significa que seamos mejores seres humanos, más honestos, compasivos, es necesario que el conocimiento tenga una dirección y aplicación moral - espiritual que promueva y logre estados de paz y armonía”. (p.49), por lo tanto, desarrollar la inteligencia espiritual dentro de la educación, lleva más allá del simple entrenamiento racional, es la base del amor universal y permite al individuo comprender el desarrollo intelectual, señalando Carreras y otros (1998), que "el concepto de paz es muy subjetivo y con variedad de significados, haciéndose distinción entre la paz 
interna que es la paz del sujeto”. (p.158), y la externa, la cual hace referencia a la paz del objeto, es decir, aquella que recae sobre un fenómeno cultural, jurídico, político y social.

Resulta conveniente, que directivos, docentes y en general, todos los miembros de la comunidad o colectivo escolar, analicen las ventajas que ofrece la paz en aspectos relacionados con el entendimiento y conocimiento entre las personas, mayor equilibrio psíquico, aceptación de las diferencias individuales (étnicas, personalidad, inteligencia), cooperación, ayuda de forma altruista, tranquilidad, quietud en la sociedad en general y respeto al medio.

\section{Desarrollo de la Atención}

De acuerdo con Gallegos (ob.cit), el siguiente principio de la inteligencia espiritual pretende el desarrollo de la atención o cultivo de la mente. Significa en el camino espiritual, que se debe practicar la atención plena y la meditación sobre los fundamentos de la atención con la finalidad de cultivar una mente iluminada”. (p.140). Asimismo, sirve para que todo el personal, se entrene en el desarrollo de la sabiduría, es decir, entender o reflexionar correctamente sobre las enseñanzas espirituales, acerca de los aprendizajes obtenidos en el medio educativo a fin de ser mejores personas y profesionales.

En relación a lo anterior, se requiere entonces hablar de la meditación, ya que es el principal agente para desarrollar la inteligencia superior, pues la meditación e inteligencia espiritual son inherentes y dependientes. Sobre este particular, Gallegos (2010) expresa que la meditación radica en "permanecer con atención plena, primero en las horas de meditación formal y luego llevando esta atención a la vida cotidiana”. (p.68).

\section{Fomento de la Felicidad}

Con respecto al principio Fomento de la Felicidad, Gallegos (ob.cit), sostiene que la inteligencia espiritual no está conectada con ninguna religión o iglesia, es una constante en la historia humana la cual trasciende la religión organizada. No se refiere a fe o creencias "es el poder para controlar y alcanzar la felicidad. Es la llave para conducir bien tu vida, poniéndote a cargo de lo que piensas, sientes y haces; tus virtudes.” (p.34). En consecuencia, implica que los directivos y docentes posean capacidad de decidir la manera 
de responder a los eventos de la vida educativa, de tal manera que puedan ser felices a pesar de las circunstancias y no a causa de ellas.

Ciertamente, la inteligencia espiritual es el nivel más alto de las inteligencias, ya que les permite ser felices, superar el sufrimiento, dar sentido a la vida; ser y gozar la libertad, actuar con responsabilidad universal, ser éticos y honestos en lo que se hace y en las relaciones establecidas así como ser compasivos con todos los miembros del colectivo escolar.

Para Aranguren (1987), la felicidad es un don, el don de la paz interior, espiritual, de la conciliación o reconciliación con todo y con todos y, para empezar y terminar, con nosotros mismos.” (p.118). Por eso, es preciso aprender a deliberar bien sobre lo conveniente, pero con la conciencia de que ser feliz es, no sólo una tarea, sino sobre todo un regalo. .

\section{Habilidades de la Inteligencia Espiritual}

Las habilidades ligadas a la inteligencia espiritual señaladas por Emmos, citado por Andrade (2011), son las siguientes:

\section{Capacidad de Trascendencia}

Para Gallegos (ob.cit), la inteligencia espiritual es la última y la más alta inteligencia con la que se resuelven problemas de sentido y valores, permitiendo insertar la vida en un contexto más amplio y profundo, es decir, en un contexto universal, definiéndola igualmente, como la capacidad que tiene un individuo de trascender, de ir más allá del materialismo.

En todo caso, el discernimiento lleva al conocimiento pleno del todo, pero además se tiene la capacidad de separar ese todo en sus partes y entender plenamente cada una de ellas de manera independiente e integrada. La inteligencia espiritual tiene la capacidad de visión cabal, de visión holística (integral) de la realidad, permiten entender contextos y totalidades, discernir la esencia última de las cosas, generando una sabiduría del mundo educativo, de las necesidades e intereses de sus miembros. 
Capacidad de utilizar recursos espirituales

Desarrollar la Inteligencia Espiritual como habilidad, requiere según expresa Gallegos (ob.cit), de un nivel de inteligencia que se genera con la capacidad de resolver problemas de significado, mostrando una conducta ética, un alto grado de discernimiento, ecuanimidad y con la capacidad de amor universal, de donde naturalmente se desprenden otras capacidades como el servir, aprender a vivir en paz y por lo tanto, se alcanza la posibilidad de ser feliz a pesar de las circunstancias. En otras palabras, se trata de la capacidad para resolver problemas que permite abordar una situación en la cual se persigue un objetivo, así como determinar el camino adecuado que conduce a dicho objetivo.

En este orden de ideas, los productos van desde teorías científicas hasta composiciones musicales, pasando por campañas políticas y de mercado exitoso, este tipo de objetivos solo tratan de las capacidades que son universales a la especie humana al afianzar el logro de las metas personales e institucionales propuestas.

\section{Comportamiento Virtuoso}

La formación debe fundamentarse en la pedagogía del amor universal; para formar seres humanos integrales en valores como la solidaridad, paz, concordia, tolerancia, paciencia, diálogo, democracia, compasión, amor, fraternidad entre otros aspectos, lo que hace un ser virtuoso y generoso.

Al respecto, Zohar y Marschall (ob.cit), afirman que las personas espiritualmente inteligentes son flexibles, poseen un nivel enaltecido de autoconocimiento, desarrollan la capacidad para afrontar al dolor y aprenden del sufrimiento, fundamentan su comportamiento en ideas y valores, se resisten e causar daño a otros y cuestionan constantemente sus acciones.

\section{Valores Personales}

La comprensión de los valores depende de quienes lo perciben, por ello, resulta difícil encontrar un concepto específico de los mismos entre los psicólogos, filósofos y sociólogos que tratan el tema, y en ese sentido, Rokeach (1973) citado por Palacios (1997), define el valor como aquellas creencias que poseen las personas acerca de estados finales y/o 
conductas deseables y que transcienden, por tanto, las situaciones concretas guiando la selección y evaluación de las situaciones y comportamientos.

Para Tomayo y Gondim (1996), los valores contribuyen a la resolución de tres cuestiones: la conciliación de intereses individuales o de grupo; la elaboración de una estructura necesaria para la organización que contemple la definición de papeles, normas, reglas para relaciones u organizaciones de trabajo, y la conciliación "entre los intereses de la organización, del medio social y natural, capaz de responder a la necesidad de la organización de producir o sobrevivir”. (p. 62).

Visto así, los valores legitiman la existencia de la organización, su estructura y su funcionamiento, incluyendo patrones de conducta de las negociaciones y las prácticas ejecutadas, en un contexto social más amplio. En consecuencia, pueden legitimar o sancionar alternativas estratégicas como deseables y aceptables, de acuerdo al contexto organizacional.

\section{Valores Terminales}

Cortina (2000), considera que los valores terminales personales “perfeccionan al hombre en lo más íntimamente de su ser, haciéndolo más humano, con mayor calidad como persona.” (p.320).

Por su parte, García y Dolan (1997) señalan “son los que aspira el individuo para sí mismo” (p.75), Los cuales, adquiere en el seno familiar y social siendo fundamentales los que se nombran a continuación:

\section{El respeto}

Es aceptar y comprender tal como son a los otros, aceptando formas de pensar diversas a la propia. De acuerdo con Carreras y otros (ob.cit), el respeto “es la consideración, atención o admiración que se le debe a una persona.” (p.199).

\section{Solidaridad}

López y Violi (ob.cit), afirman que es “el valor que facilita la capacidad para adherirse a la empresa de otros; es sentir, expresar y actuar de tal manera, que las personas sepan que 
pueden contar con su apoyo.” (p.79). Esto significa ponerse en el puesto del otro.

\section{Armonía Interior}

Para Palacios (Ob.cit), significa "libre de conflicto interno". Es la conjunción entre el movimiento, el orden, la estabilidad y el equilibrio que se percibe en un objeto real o imaginario, lo cual estimula al sujeto, produciéndole una agradable sensación de paz, alegría o éxtasis interior.

\section{Tolerancia}

Implica una actitud comprensiva frente a las opiniones del otro, sin la cual se hace imposible establecer relaciones armónicas, manifestar el reconocimiento de la dignidad del otro. Para Ortega y otros (2003), “está determinada por creencias básicas que orientan la propia vida del ser humano, no se hereda, se aprende.” (p.57).

\section{Cooperación}

Parte del trabajo en equipo, propio de las sociedades democráticas. Significa, según Londoño (2002), que cada persona “debe hacer aportes útiles a los demás integrantes del grupo para que todos se benefician de ello.” (p.400).

\section{Justicia}

La justicia como valor y la injusticia como contravalor aparecen en diversas formas durante la convivencia entre los seres humanos. Según Carreras y otros (Ob.cit), se define como “actitud moral o voluntad decidida de dar a cada uno lo que es suyo.” (p.11).

\section{Libertad}

Para Palacios (Ob.cit), este valor clasificado como ético- social se relaciona con la independencia, la libre elección. De modo semejante, cuando Guardini (1982), analiza el fenómeno vital del acto libre, distingue la libertad como la que se manifiesta como expresión libre del ser íntimo. 


\section{Valores Instrumentales}

Para Rokeach, citado por García y Dolan (Ob.cit), estos valores “se encargan de guiar al sujeto hacia un comportamiento responsable, honestidad, lealtad, convivencia, entre otros”. (p.74) Representan métodos de conducta para alcanzar los valores finales. Visto así, estos valores ayudan a sentir deseosos de proyectar acciones de convivencia y honestidad, relacionándolos con el ámbito educativo, al sentir necesidad de contar con los otros.

Según García y Dolan (Ob.cit) los valores “conducen al individuo hacia el comportamiento lógico, imaginario e inteligente, profesión, dinero, trabajo, así mismo y a otros. Estos tienden a crear una disposición al comportamiento competente y su violación lleva a comportamientos de tristeza e inadecuación y conflictos”. (p.74). Sirven entonces, para adaptarse y sobrevivir, no están sujetos a la moral. Entre éstos destacan:

\section{Honestidad}

Aguilar (2002), señala que las influencias a las que están expuestos los individuos han aumentado en los últimos años. Ahora, no sólo la familia, la escuela y la iglesia son los encargados de la transmisión de los valores, las creencias o las tradiciones; también los medios de comunicación y los avances tecnológicos le ofrecen "todo un universo que en cierta medida refuerza los valores personales, pero que al mismo tiempo, genera nuevos modos de comportarse, creer y sentir dentro de la sociedad.” (p.62). En consecuencia, cuando se piensa de una manera y se termina obrando de otra, o cuando se actúa correctamente con la aprobación de los demás pero no lo siente de verdad en el corazón, no se está viviendo a plenitud el valor de la honestidad, aspecto que podría conducir a la aparición de sus contravalores.

\section{Responsabilidad}

Faría (2001), expresa, la responsabilidad es la "capacidad que tiene el ser humano de conocer y aceptar (individual) e influir (colectiva) en las decisiones y actos que realiza conscientemente.” (p.101) Por lo tanto, este valor ético-social debe estar incorporado en cada proyecto personal, porque el ser humano no puede vivir sin metas definidas. 


\section{Lealtad}

Para Rokeach (1973) citado por Palacios (ob.cit), es un valor clasificado dentro de la categoría de valores instrumentales ético- morales. Es un sentimiento físico y mental hacia otro. Implica una noción abstracta de compromiso con ciertos valores morales y éticos. Es una virtud humana que tiene como objetivo facilitar el establecimiento de profundos lazos de amor, solidaridad y cariño entre los individuos que forman una comunidad.

\section{Convivencia}

Según Gil (2004), la educación “está directamente relacionada con la convivencia, ya que todas las sociedades están rodeadas por fuerzas disgregadoras y fuerzas convivenciales, es decir, no se encuentran en la vida al cincuenta por ciento, pues siempre una supera a la otra” (p.89). Cuando se da un desequilibro se buscan las causas del mismo y esto acaba confundiendo al individuo al llegar a diversas conclusiones quizás inadecuadas al problema presentado.

\section{Lógica}

Para García y Pérez (2005), “la estructura de la lógica es el aspecto formal de los estadios de razonamiento moral, la argumentación racional del mismo o razonamiento que la persona elabora para justificar una determinada decisión moral;” (p. 133), es decir, es el modo cualitativo de pensamiento que subyace tras el contenido moral o modo lógico de organizar y expresar el mismo y que atiende a la estructura organizable de la justicia, siendo definido como valioso en relación con los valores morales y por la razón de la elección axiológica; es universal y supone el avance del desarrollo moral, a través de sucesivas transformaciones de la estructura cognitiva de la persona.

\section{Inteligencia}

Gardner citado por Goleman (2004), señalaba que las personas tienen siete tipos de inteligencia. La lingüística, inteligencia relacionada con la capacidad verbal, con el lenguaje y con las palabras; la lógica, asociada con el desarrollo del pensamiento abstracto. 
Es la capacidad de relacionar conocimientos que se poseen para resolver una determinada situación. Consiste en saber elegir la mejor opción para resolver un problema.

\section{Trabajo en Equipo}

La integración del trabajo en equipo en el medio escolar requiere una planeación cuidadosa, ningún equipo puede surgir por sí mismo, se asume por tanto, que el director debe trabajar con los docentes para comunicar y explicar algunos factores: cómo espera que trabajen, qué difiere el nuevo método de funcionamiento respecto al que estaban acostumbrados, dónde pueden solicitar ayuda y cómo funcionará el trabajo en equipo para la toma de decisiones, entre otros aspectos.

En relación a lo expuesto, Hughes y otros (2007), señalan que pocas actividades se han vuelto tan populares en las organizaciones en estos días que los talleres para la formación de equipos "una razón para este nivel de actividad es el cambio importante que se ha presentado en el ambiente laboral desde un enfoque primario individual hacia un trabajo centrado en el equipo”. (p.502).

\section{ASPECTOS METODOLÓGICOS}

La presente investigación es de tipo proyectiva, ya que tuvo como objetivo central proponer lineamientos estratégicos para fortalecer la inteligencia espiritual y los valores personales en los integrantes de la Coordinación del Proyecto Educativo Regional; al referir en su propósito general una propuesta de lineamientos estratégicos; siguiendo el planteamiento de Hurtado (2010), en cuanto a la finalidad de las investigaciones proyectivas es proponer soluciones a la situación planteada. En este sentido, de acuerdo con el nivel de profundidad, es descriptiva correlacional, según Chávez (2007), “describe y establece la relación entre las variables del estudio, mide sin realizar inferencias ni verificar hipótesis” (p.135); en consecuencia, es descriptiva correlacional por cuanto se parte de la observación y descripción de los hechos que se refieren a las variables objeto de estudio, tal y como se presentan en la realidad

Por consiguiente, esta investigación se ubicó en el paradigma positivista, específicamente al método utilizado es el empirista-inductivo, de acuerdo con Vieytes (2004), “el enfoque cuantitativo reposa en el método matemático para cuantificar las variables” (p. 63). Desde la perspectiva más general, según la fundamentación empírica, solo en los hechos puede 
encontrarse la verdad; tal como lo expone Barrera (2008), en el empirismo "la experiencia es garantía de validez del conocimiento” (p.55), a tal efecto, la manera como se desarrollan los hechos y la forma de participar en ellos constituye la verdadera fuente del saber.

Significa entonces, que el método inductivo en opinión de Hurtado y Toro (2007), "permite pasar de los hechos particulares a los principios generales” (p.57). En concordancia con lo expuesto, la inducción permite al investigador llegar lógicamente al entendimiento desde el conocimiento de los fenómenos o hechos, a la ley o principio que enmarca a todos ellos de manera uniforme; lo cual se conoce como generalización inductiva, que sustenta el carácter nomotético del positivismo.

El diseño de este estudio es considerado como no experimental, transeccional y de campo; de acuerdo con Hernández, Fernández y Baptista (2008), se observaron los fenómenos tal y como ocurren en su contexto natural, para después analizarlos; no se construyó ninguna situación, sino que se observaron las existentes y no se provocaron intencionalmente por quien investigó, es transeccional por que los datos se recogieron en un único momento y tiempo, es de campo por cuanto los datos se recolectaron en las instituciones educativas del Municipio Santa Rita, donde se analizó y describió específicamente la Inteligencia Espiritual y los Valores Personales en el personal de dichas instituciones.

En relación a la población está constituida por la totalidad de los sujetos hacia los cuales se orientó la aplicación del objeto de la investigación, por lo que, constituye un censo poblacional, conformada por 166 sujetos, distribuidos en cargos de la siguiente manera: (16) directivos, (95) docentes (9), Administrativos y (46) obreros de las Instituciones Educativas pertenecientes a la Secretaria de Educación, Coordinación del PER en el Municipio Santa Rita, Estado Zulia. En esta investigación se utilizó como técnica la encuesta, y se empleó como instrumento de recolección de datos un cuestionario elaborado por la investigadora; dirigido al personal directivo, docente, administrativo y obrero, de las escuelas objeto de estudio.

El instrumento de recolección de datos (cuestionario), elaborado está constituido por (44) ítems cada uno con cinco (05) alternativas de respuestas: Siempre (5), Casi Siempre (4), Algunas Veces (3), Casi Nunca (2), Nunca (1). Con el mismo se midieron las variables del estudio: Inteligencia Espiritual y Valores Personales.

Para realizar el procesamiento y análisis estadístico de los resultados arrojados a través 
de la aplicación del cuestionario, orientado al logro de los objetivos específicos 1, 2 y 3, se utilizó la estadística descriptiva, el cual permitió la descripción de las variables de estudio específicamente a través del uso de la media y la desviación estándar. En este orden de ideas, la utilización de la media permitió la categorización de ítemes, indicadores, dimensiones, así como de las variables en estudio. Asimismo, para la presentación de los resultados, se diseñaron una serie de tablas por variable, dimensiones e indicadores, que muestran las medidas utilizadas para los fines del análisis con sus respectivos valores arrojados a través del procesamiento de datos.

Para el establecimiento de la relación entre las variables, se recurrió al coeficiente estadístico de correlación de Spearman. Este coeficiente estadístico de correlación, se hizo a través de la aplicación del software SPSS, el cual permitió obtener el valor respectivo.

\section{RESULTADOS}

En cuanto a la variable Inteligencia Espiritual, se evidencia un promedio para las respuestas de los directivos de 3.32, con una muy baja dispersión de 0.73; para los docentes de 3.15 y 0.73; en cuanto a los obreros y administrativos de 3.03; 3.24 y su dispersión de 0.69 y 0.77. En consecuencia, se consideran los principios y las habilidades de la inteligencia espiritual, logrando fortalecer su espiritualidad sobre la base de las actividades que ejecutan, logrando generar respuestas afectivas agradables en el colectivo escolar; así como crear un clima de cordialidad ante situaciones desfavorables, debido a que, ante una disputa, se toma en consideración los planteamientos del otro para buscar una solución efectiva.

Asimismo, se evidencia capacidad para entender a los otros, dando respuesta al por qué de las situaciones con ecuanimidad, al tiempo de reflejar un comportamiento virtuoso (entendido como: tolerante, paciente, democrático) le permite mejorar las relaciones interpersonales; además, cumplen las promesas que hace ante su equipo de trabajo. 


\section{Tabla 1}

\section{Variable. Inteligencia Espiritual}

\begin{tabular}{|l|c|c|c|c|c|c|c|c|c|}
\hline \multicolumn{2}{|c|}{ Dimensiones } & \multicolumn{2}{c|}{ Directivos } & \multicolumn{2}{c|}{ Docentes } & \multicolumn{2}{c|}{ Obreros } & \multicolumn{2}{c|}{$\begin{array}{c}\text { Administrativ } \\
\text { os }\end{array}$} \\
\cline { 3 - 11 } & $\mathbf{X}$ & $\mathbf{S}$ & $\mathbf{X}$ & $\mathbf{S}$ & $\mathbf{X}$ & $\mathbf{S}$ & $\mathbf{X}$ & $\mathbf{S}$ \\
\hline $\begin{array}{l}\text { Principios de la inteligencia } \\
\text { espiritual }\end{array}$ & 3,29 & 0,80 & 3,01 & 0,82 & 2,82 & 0,81 & 3,10 & 0,82 \\
\hline $\begin{array}{l}\text { Habilidades de la inteligencia } \\
\text { espiritual }\end{array}$ & 3,35 & 0,66 & 3,30 & 0,64 & 3,23 & 0,58 & 3,39 & 0,72 \\
\hline Promedio general & $\mathbf{3 , 3 2}$ & $\mathbf{0 , 7 3}$ & $\mathbf{3 , 1 5}$ & $\mathbf{0 , 7 3}$ & $\mathbf{3 , 0 3}$ & $\mathbf{0 , 6 9}$ & $\mathbf{3 , 2 4}$ & $\mathbf{0 , 7 7}$ \\
\hline
\end{tabular}

En cuanto a la variable valores personales, el personal considera dentro de sus acciones valores terminales e instrumentales que contribuyen al fortalecimiento de su trabajo sobre la base de criterios éticos claramente definidos: respeto, solidaridad, armonía interior, tolerancia, cooperación, justicia, así como libertad, ajustados a los requerimientos sociales.

Asimismo, se acepta la forma de pensar de sus compañeros y se promueven sentimientos de compartir obligaciones con los miembros del plantel; asimismo, ayudan a los compañeros que manifiesten necesidades. Al estar libre de conflictos internos, puede ayudar a mejorar a sus compañeros de trabajo; asimismo, fomenta la práctica de dar y recibir en sus relaciones con el personal. Igualmente, se conoció que directivos y docentes son equitativos a la hora de distribuir las responsabilidades en un trabajo en conjunto; asimismo, al cumplir con sus obligaciones laborales toman en cuenta los lineamientos de la organización, siendo equitativos al momento de distribuir las responsabilidades en un trabajo en conjunto.

En la dimensión valores instrumentales, se evidenció el desarrollo de acciones sobre la base de la honestidad, responsabilidad, lealtad, convivencia, lógica, inteligencia, trabajo en equipo, contribuyendo así con el fortalecimiento de valores personales instrumentales. Ante cualquier situación, asume las consecuencias de sus propios actos con lealtad hacia los miembros de las instituciones analizadas y promoviendo las actividades de convivencia para mejorar las relaciones interpersonales.

Por otra parte, el personal es capaz de manejar los conflictos surgidos en la organización; utilizando el conocimiento que posee para solucionar una determinada situación, ejecutando sus labores estableciendo una buena interacción entre los integrantes de la escuela; asimismo, actúan en conjunto para lograr un desempeño elevado. 


\section{Tabla 2}

\section{Variable. Valores Personales}

\begin{tabular}{|l|c|c|c|c|c|c|c|c|}
\hline \multirow{2}{*}{ Dimensiones } & \multicolumn{2}{|c|}{ Directivos } & \multicolumn{2}{c|}{ Docentes } & \multicolumn{2}{c|}{ Obreros } & \multicolumn{2}{c|}{$\begin{array}{c}\text { Administra } \\
\text { tivos }\end{array}$} \\
\cline { 2 - 9 } & $\mathbf{X}$ & $\mathbf{S}$ & $\mathbf{X}$ & $\mathbf{S}$ & $\mathbf{X}$ & $\mathbf{S}$ & $\mathbf{X}$ & $\mathbf{S}$ \\
\hline Valores terminales & 3,51 & 0,73 & 3,37 & 0,66 & 3,30 & 0,60 & 3,41 & 0,64 \\
\hline Valores instrumentales & 3,38 & 0,61 & 3,33 & 0,53 & 3,30 & 0,52 & 3,54 & 0,62 \\
\hline Promedio general & $\mathbf{3 , 4 4}$ & $\mathbf{0 , 6 7}$ & $\mathbf{3 , 3 5}$ & $\mathbf{0 , 5 9}$ & $\mathbf{3 , 3 0}$ & $\mathbf{0 , 5 6}$ & $\mathbf{3 , 4 8}$ & $\mathbf{0 , 6 3}$ \\
\hline
\end{tabular}

Fuente: Elaboración propia

En cuanto al objetivo, que estuvo orientado en establecer la relación entre la inteligencia espiritual y los valores personales de los integrantes de la Coordinación del Proyecto Educativo Regional en el municipio Santa Rita, se presentan los resultados de acuerdo a la aplicación de la fórmula Rho, arrojando los siguientes valores:

\section{Tabla 3}

\section{Resultados de la correlación entre las variables (Rho)}

\begin{tabular}{|c|c|c|c|c|}
\hline $\begin{array}{c}\text { Variable1: Inteligencia } \\
\text { espiritual }\end{array}$ & Directivos & Docentes & Obreros & Administrativos \\
\hline $\begin{array}{c}\text { Variable 2: Valores } \\
\text { personales }\end{array}$ & $0.72 * *$ & $0.75 * *$ & $0.77 * *$ & $0.70 * *$ \\
\hline
\end{tabular}

De acuerdo con los resultados obtenidos, se arrojó para las cuatro fuentes de información una correlación positiva fuerte, lo cual indica que la inteligencia espiritual es determinante de los valores personales, logrando fortalecer el respeto, solidaridad, armonía interior, cooperación, así como la responsabilidad, lealtad, convivencia, inteligencia, trabajo en equipo, situación que proporciona las bases necesarias para el desarrollo de sus acciones ajustados a comportamiento ético de la sociedad.

\section{LINEAMIENTOS}

\section{Justificación}

Los resultados obtenidos en las variables inteligencia espiritual y valores personales, se ubicó en la categoría moderada, indica que en ese mismo nivel los directivos, docentes, obreros y administrativos, que fue la fuente de información, por lo que es necesario 
promover acciones tendentes a practicar en las relaciones interpersonales a fin de mantener un equilibrio en la organización a fin de mejorar el desempeño.

De igual manera, al correlacionar los resultados de las dos variables, se obtuvo una correlación positiva fuerte, lo cual indica que la inteligencia espiritual es determinante de los valores personales, logrando fortalecer el respeto, solidaridad, armonía interior, cooperación, así como la responsabilidad, lealtad, convivencia, inteligencia, trabajo en equipo, situación que proporciona las bases necesarias para el desarrollo de sus acciones ajustados a comportamiento ético de la sociedad.

En consecuencia, se consideran los principios y las habilidades de la inteligencia espiritual, a fin de fortalecer su espiritualidad sobre la base de las actividades que ejecutan, logrando generar respuestas afectivas agradables en el colectivo escolar; así como crear un clima de cordialidad ante situaciones desfavorables, debido a que, ante una disputa, se toma en consideración los planteamientos del otro para buscar una solución efectiva.

En ese sentido, los resultados obtenidos en el estudio, justifican la elaboración de lineamientos con base en los seis caminos de Zohar y Marshall (ob. cit), como: el deber, amor y entrega, conocimiento, hermandad, entre otros, que brindan bases sólidas para que las actividades personales y profesionales se realicen en atención a las necesidades.

\section{Objetivo}

Proporcionar una orientación teórica - conceptual y práctica para el fortalecimiento de los valores personales sobre la base de su inteligencia espiritual, logrando afianzar sus principios éticos en consonancia con sus acciones personales y/o profesionales; considerando los seis caminos de Zohar y Marshall (ob. cit), como: el deber, amor y entrega, conocimiento, hermandad, entre otros, que brindan bases sólidas para que actividades personales y profesionales se realicen en atención a las necesidades

\section{El Camino del deber}

Se refiere a la pertenencia, la cooperación, la contribución y el sustento en el seno de la comunidad. La seguridad y la estabilidad dependen de la experiencia con los demás y con el entorno, normalmente desde la infancia, a fin de seguir la senda del deber de un modo 
espiritualmente inteligente, las personas deben querer pertenecer a su grupo, asumir un compromiso interior con su pertenencia a él y comprender por qué lo hace.

\section{El Camino del amor y de la entrega}

Se refiere a amar, nutrir, proteger y fertilizar. Dentro del contexto educativo, este camino implica fortalecer sus acciones sobre la base de las necesidades del entorno, logrando alcanzar cada uno de los objetivos establecidos.

\section{El Camino del conocimiento}

Abarca desde comprender problemas prácticos generales, la búsqueda filosófica de la verdad, la búsqueda espiritual de Dios y su Voluntad hasta la unión definitiva con Él, por medio del conocimiento.

\section{El Camino de la transformación personal}

Es el más relacionado con el punto divino de actividad cerebral, con las personalidades más abiertas a las experiencias místicas, con las emociones más extremas, con quienes son tildados de «excéntricos» o diferentes y con quienes a menudo deben batallar por su salud mental (y a veces perder).

\section{El Camino de la hermandad}

Permite superar el miedo a los cambios, donde el alma individual es y siempre será parte de la mayor y eterna alma del mundo, por ello, son especialmente esas cualidades del alma que se desarrollan en el camino de la hermandad y luego en el consiguiente camino del liderazgo de servicio las que permanecen eternas.

\section{El Camino del liderazgo con voluntad del servicio}

Para ser un líder eficaz, un individuo normalmente debe tener las maneras abiertas y seguras del tipo de personalidad emprendedora. 


\section{CONCLUSIONES}

Los resultados obtenidos en el estudio, permiten proponer lineamientos estratégicos para fortalecer la inteligencia espiritual y los valores personales de los directivos, docentes, administrativos y obreros en la Coordinación del Proyecto Educativo Regional en el Municipio Santa Rita, pues existe una moderada aplicación de los principios de la inteligencia espiritual, los cuales se concretan en la fortaleza del amor; promoción del bien; actitud hacia la paz; desarrollo de la atención; fomento la felicidad, contribuyendo en cierto sentido hacia alcanzar una inteligencia espiritual que contribuya con su crecimiento personal y profesional dentro de las instituciones analizadas.

Considerando que las habilidades de la inteligencia espiritual constituyen la capacidad de trascendencia y la capacidad en el uso de recursos espirituales para solucionar problemas, se ubicaron en moderada presencia.

En el mismo sentido, los valores terminales se ubicaron en moderada presencia, lo que es importante porque permiten laborar sobre la base de criterios de respeto, solidaridad, armonía interior, tolerancia, cooperación, justicia y libertad, sustentados en sus valores terminales, generando acciones en consonancia con los parámetros sociales.

Los valores instrumentales, se obtuvo moderada presencia, pues la honestidad, responsabilidad, lealtad, convivencia, lógica, inteligencia y trabajo en equipo, se deben fortalecer sobre la base de sus actividades tanto personales como dentro de las escuelas para garantizar la eficiencia en el logro de sus objetivos.

\section{RECOMENDACIONES}

En consonancia con las conclusiones emitidas, es pertinente recomendar:

Al Departamento de Desarrollo General Académico de la Secretaría de Educación, evalúe la manera como desarrolla el personal de estudio su inteligencia espiritual, dado que quienes la poseen lograr generar un elevado nivel de autoconocimiento, con capacidad para enfrentar el dolor y aprender con el sufrimiento, es decir, ser resilientes, por tanto, es fundamental proporcionarle ideas, con capacidad de rechazar aquellas situaciones que les hacen daño, logrando fortalecer su carácter para alcanzar sus objetivos. 
A los coordinadores académicos que fomenten la inteligencia espiritual, pues aporta beneficios que contribuyen a sobrellevar el estrés, el consumismo, el ego, y la necesidad de lo material, brindándole un mejor sentido de la vida, por tanto, se requiere la formulación de talleres prácticos que orienten al personal en aprovechar la vida aún más desde la perspectiva emocional, espiritual, dejando un poco de lado lo material y superficial.

El desarrollo de la inteligencia espiritual, contribuye con la compresión y aceptación de uno mismo como una persona que vale, que tiene misiones definidas dentro de una sociedad, y que se conecta con las carencias y fortalezas personales, por ello, se recomienda a la Coordinación del PER que se le proporcione al personal directivo, docente, administrativo y obrero, una guía para que conozcan los beneficios de la parte espiritual.

Las personas que desarrollan su inteligencia espiritual descubren un nuevo lenguaje de comunicación, se dan cuenta que la vida les proporciona un sentido más profundo y trascendente, contactándose con sus valores, reconociendo la importancia de sus sentimientos y pensamientos, así como en el impacto que tienen en su entorno; por tanto, se recomienda a los integrantes de las instituciones educativas, que evalúen su inteligencia emocional y espiritual, logrando que los mismos puedan generar a corto plazo el fortalecimiento de la inteligencia espiritual.

Los valores constituyen una herramienta que se asume desde la familia, por tanto, se recomienda al Proyecto Educativo Regional realice una evaluación de los valores tanto terminales como instrumentales del personal, lo cual permitirá fortalecerlos sobre la base de sus necesidades.

A la Secretaría de Educación propiciar talleres basados en la formación en valores, logrando que todo el personal participe directamente para conocer sus beneficios, lo que implica laborar sobre la base de criterios éticos claramente definidos, convirtiéndose en un modelo a seguir para el desarrollo social.

A los directores de las instituciones educativas, considerar los lineamientos planteados, logrando que todo el personal tenga la capacidad de seguir los caminos planteados por Zohar y Marshall (ob. cit) logrando que puedan fortalecer su inteligencia espiritual en consonancia con criterios basados en valores. 


\section{REFERENCIAS}

Aguilar, L. (2002). Desarrollo de los valores en el ámbito educativo. Ediciones Fe y Alegría. Caracas. Venezuela.

Andrade, H. (2011). Cambio y Fuera. Dirigir en el siglo XXI. Palibrio. EE.UU.

Aranguren, J. (1987). Moral de la vida cotidiana, personal y religiosa. Editorial Tecnos. Madrid.

Barrera M. (2008). Modelos epistémicos en investigación y educación. Quinta edición. Ediciones Quirón Sypal. Caracas Venezuela.

Barroso, C. (2005). Valores de la organización. Trillas. México.

Buzan, T. (2005). Poder de la inteligencia espiritual. Ediciones Urano. España.

Carreras, LL; Eijo P;Estany A; Gómez M; Guich R; Mir V; Ojeda F; Planas T;Serrats M. (1998). Como educar en valores. Narcea S. A. Ediciones 6ta edición. Madrid. España.

Chávez, N. (2007). Introducción a la Investigación Educativa. 2da Edición, Taller Arts Grafica, S.A. Maracaibo Venezuela.

Cortina, A (2000). El mundo de los valores. Editorial El Búho. Colombia.

Covey, S. (2005). El 8vo hábito. De la efectividad a la grandeza. Editorial Planeta colombiana. Colombia.

Faria, J. (2001). Filosofía, ética y moral. Santa fe de Bogotá, Colombia.

Gallegos, R. (2005). “Educación y Espiritualidad, La educación como practica espiritual”. Ed. Fundación Internacional para la Educación Holista. Guadalajara, Fragmento publicado en: www.ramongallegos.com.

Gallegos, R. (2006). Inteligencia Espiritual. Más allá de las inteligencias múltiples y emocional Guadalajara. México.

Gallegos, R. (2007). Inteligencia espiritual. Más allá de las inteligencias múltiples y emocional. Fundación Internacional para la Educación Holista, Guadalajara.

Gallegos, R. (2010). La Conciencia Iluminada. Trilogía de inteligencia espiritual II. Fundación Internacional para la Educación Holista, Guadalajara.

García, J. y Pérez, E. (2005). Razonamiento moral y valores: estudio de sus relaciones en un grupo de universitarios españoles. Revista Latinoamericana de psicología. 
[Revista en línea] Vol. 37, número 1, $2005 . \quad$ Disponible: http://www.redalyc.org/pdf/805/80537108.pdf [Consulta: 2012, septiembre, 05].

García,S. y Dolan, S. (1997). Dirección por valores. Editorial Mc Graw-Hill. España.

Gil, R (2004). La educación en valores. Paidós. Barcelona.

Goleman, D. (2004). Inteligencia emocional. Editorial Kairós. Argentina

Guardini, R. (1982). Cristianismo y sociedad. Salamanca: Sígueme. pp. 75-76

Hernández, R.; Fernández, D. y Baptista, P. (2008). Metodología de la Investigación. 4ta Edición. McGraw-Hill Interamericana. México

Hughes, R. Ginnett, R., y Curphy, G. (2007) Liderazgo. Cómo aprovechar las lecciones de la experiencia. Mc Graw Hill. México.

Hurtado de Barrera, J (2010). Metodología de la investigación Holística. Fundación Sypal. Caracas Venezuela.

Hurtado I, y Toro J. (2007). Paradigmas y métodos de investigación en tiempos de cambio. Episteme Consultores Asociados C. A. Segunda Edición. Caracas Venezuela.

Londoño, L (2002). ¿Cómo utilizar nuestros valores? Editorial Trillas. México.

López, M y Violi, D (2001). Cómo criar niños en tiempos difíciles. Editorial Norma. Bogotá Colombia.

Millman, D. (1995). Inteligencia espiritual. Swami ediciones. España.

Ortega, R.; Del Rey y Fernández, I. (2003). Investigación educativa e Intervención contra la Violencia Escolar en España. Machado Libros. Madrid España.

Palacios, S. (1997). Los valores humanos y la comprensión de la desobediencia civil. Revista de psicodidáctica N.3. Universidad de la Rioja. España.

Ramos, M. (2005). Para educar en valores. Teoría y práctica. Grupo Editorial Latinoamericano. El Hatillo. Edo. Miranda. Venezuela

Rodríguez, F. (2012). VIII Congreso. Ciencia y Espiritualidad. Aso. UNESCO para el diálogo de Malaga. España. Disponible en http://unesco-malaga.blogspot.com/

Tomayo, Á y Gondim, M. (1996). Escala de valores organizacionales. Revista de Administración. Vol. 31, nº 2. Pp. 62-72 San Pablo. Brasil 
Inteligencia Espiritual y Valores Personales en los integrantes de la Coordinación del

Verdú, V. (2008). La Producción del Bien. [Documento en línea] Disponible en http://www.elboomeran.com/blog-post/11/5191/vicente-verdu/la-produccion-delbien/ [Consulta: 2012, junio 08]

Vieytes Ruth. (2004). Metodología de la investigación en organizaciones, mercado y sociedad. Epistemología y técnicas. Editorial de las Ciencias. Argentina

Zohar, D., y Marshall, I. (2001). La inteligencia espiritual. La inteligencia que permite ser creativo, tener valores y fe. Plaza \& Janés Editores. Madrid - España. 